\title{
Compact conformally Kähler Einstein-Weyl manifolds
}

\author{
Włodzimierz Jelonek
}

Received: 20 November 2011 / Accepted: 27 April 2012 / Published online: 25 May 2012

(C) The Author(s) 2012. This article is published with open access at Springerlink.com

\begin{abstract}
We give a description of compact conformally Kähler Einstein-Weyl manifolds whose Ricci tensor is Hermitian.
\end{abstract}

\section{O Introduction}

In this paper we shall investigate compact Einstein-Weyl structures $(M,[g], D)$ on a complex manifold $(M, J), \operatorname{dim} M \geq 4$, which are conformally Kähler and whose Ricci tensor $\rho^{D}$ is Hermitian, i.e., $\rho^{D}$ is $J$-invariant.

We give a complete classification of compact Einstein-Weyl structures $(M,[g], D)$ with $\operatorname{dim} M \geq 4$ such that $(M,[g], J)$ is conformally Kähler, i.e., there exists a metric $g_{0} \in[g]$ such that $\left(M, g_{0}, J\right)$ is Kähler and whose Ricci tensor $\rho^{D}$ is $J$-invariant, i.e.,

$$
\rho^{D}(J X, J Y)=\rho^{D}(X, Y) \text {. }
$$

Conformally Kähler Einstein manifolds were classified by Derdziński and Maschler in [4]. Compact Gray bi-Hermitian manifolds are partially classified in [10]. It is proved in [9] that compact Einstein-Weyl manifolds are also Gray manifolds (see [8]). The compact EinsteinWeyl 3-D manifolds are studied in [17]. The compact Einstein-Weyl manifolds on complex manifolds compatible with complex structure are studied in $[15,16,13,18]$. In [6] there are studied Riemannian manifolds $(M, g)$ in four dimensions which are locally conformally Kähler. The Einstein-Weyl conformally Kähler structures studied in $[13,18]$ have the $J$-invariant Ricci tensor $\rho^{D}$ of the Weyl structure $(M,[g], D)$. In the first section of the paper we recall some facts from [9] and describe compact Einstein-Weyl manifolds with the Gauduchon metric as Gray manifolds. In Sect. 2 we describe the Riemannian structure of Gray manifold corresponding to Einstein-Weyl manifold with the Gauduchon metric. In Sect. 3 we prove that compact Einstein-Weyl manifold with Hermitian Ricci tensor admits a holomorphic Killing

W. Jelonek $(\varangle)$

Institute of Mathematics, Cracow University of Technology,

Warszawska 24, 31-155 Kraków, Poland

e-mail: wjelon@pk.edu.pl 
vector field with special Kähler-Ricci potential and consequently $M=\mathbb{C P}^{n}$ or $M=\mathbb{P}(L \oplus \mathcal{O})$ where $L$ is a holomorphic line bundle over Kähler-Einstein manifold. In Sect. 4 we prove that if $M=\mathbb{P}(L \oplus \mathcal{O})$ then $L$ is a holomorphic line bundle over Kähler-Einstein manifold with positive scalar curvature.

\section{Einstein-Weyl geometry and Killing tensors}

We start with some basic facts concerning Einstein-Weyl geometry. For more details see $[17,15,16]$.

Let $M$ be a $n$-dimensional manifold with a conformal structure $[g]$ and a torsion-free affine connection $D$. This defines an Einstein-Weyl (E-W) structure if $D$ preserves the conformal structure, i.e., there exists a 1 -form $\omega$ on $M$ such that

$$
D g=\omega \otimes g
$$

and the Ricci tensor $\rho^{D}$ of $D$ satisfies the condition

$$
\rho^{D}(X, Y)+\rho^{D}(Y, X)=\bar{\Lambda} g(X, Y) \text { for every } X, Y \in T M
$$

for some function $\bar{\Lambda} \in C^{\infty}(M)$. Gauduchon proved ([7]) the fundamental theorem that if $M$ is compact then there exists a Riemannian metric $g_{0} \in[g]$ for which $\delta \omega_{0}=0$ and $g_{0}$ is unique up to homothety. We shall call $g_{0}$ a standard metric of E-W structure $(M,[g], D)$. Let $\rho$ be the Ricci tensor of $(M, g)$ and let us denote by $S$ the Ricci endomorphism of $(M, g)$, i.e., $\rho(X, Y)=g(X, S Y)$. We recall two important theorems (see $[17,15])$ :

Theorem 1.1 A metric $g$ and a 1-form $\omega$ determine an E-W structure if and only if there exists a function $\Lambda \in C^{\infty}(M)$ such that

$$
\rho^{\nabla}+\frac{1}{4}(n-2) \mathcal{D} \omega=\Lambda g
$$

where $\mathcal{D} \omega(X, Y)=\left(\nabla_{X} \omega\right) Y+\left(\nabla_{Y} \omega\right) X+\omega(X) \omega(Y)$ and $n=\operatorname{dim} M$. If (1.2) holds then

$$
\bar{\Lambda}=2 \Lambda+\operatorname{div} \omega-\frac{1}{2}(n-2)\left\|\omega^{\sharp}\right\|^{2}
$$

Tod proved [17] that the Gauduchon metric admits a Killing vector field, more precisely he proved:

Theorem 1.2 Let $M$ be a compact $E$-W manifold and let $g$ be the standard metric with the corresponding 1-form $\omega$. Then the vector field $\omega^{\sharp}$ dual to the form $\omega$ is a Killing vector field on $M$.

By $\tau=\operatorname{tr}_{g} \rho$ we shall denote the scalar curvature of $(M, g)$. Compact E-W manifolds with the Gauduchon metric are Gray manifolds. To define Gray manifolds we define first a Killing tensor.

Definition A self-adjoint $(1,1)$ tensor on a Riemannian manifold $(M, g)$ is called a Killing tensor if

$$
g(\nabla S(X, X), X)=0
$$

for arbitrary $X \in T M$. 
Remark The condition $g(\nabla S(X, X), X)=0$ is equivalent to

$$
\mathfrak{C}_{X, Y, Z} g(\nabla S(X, Y), Z)=0
$$

for arbitrary $X, Y, Z \in \mathfrak{X}(M)$, where $\mathfrak{C}$ denotes the cyclic sum.

Now we can give

Definition A Riemannian manifold $(M, g)$ will be called a Gray $\mathcal{A} \oplus \mathcal{C}^{\perp}$ manifold if the tensor $\rho-\frac{2 \tau}{n+2} g$ is a Killing tensor.

In this paper, Gray $\mathcal{A} \oplus \mathcal{C}^{\perp}$ manifolds will be called for short Gray manifolds or $\mathcal{A} \oplus \mathcal{C}^{\perp}$ manifolds. Gray manifolds were first defined by Gray ([8]).

In the next two theorems, which are proved also in [9], we characterize compact E-W manifolds $(M, g)$ with the Gauduchon metric as Gray ([8]) manifolds and show that the eigenvalues $\lambda_{0}, \lambda_{1}$ of the Ricci tensor satisfy the equation $(n-4) \lambda_{1}+2 \lambda_{0}=C_{0}=$ const which we shall use later. We sketch the proofs of the theorems. Our motivation is to use the structure of Gray manifolds to use ideas from [10], where we classified a different class of Gray manifolds. From the above theorems it follows (see [9])

Theorem 1.3 Let $(M,[g])$ be a compact $E$-W manifold, $n=\operatorname{dim} M \geq 3$, and let $g$ be the standard metric on $M$. Then $(M, g)$ is an $\mathcal{A} \oplus \mathcal{C}^{\perp}$-manifold. The manifold $(M, g)$ is Einstein or the Ricci tensor $\rho^{\nabla}$ of $(M, g)$ has exactly two eigenfunctions $\lambda_{0} \in C^{\infty}(M), \lambda_{1}=\Lambda$ satisfying the following conditions:

(a) $(n-4) \lambda_{1}+2 \lambda_{0}=C_{0}=$ const

(b) $\lambda_{0} \leq \lambda_{1}$ on $M$

(c) $\operatorname{dim} \operatorname{ker}\left(S-\lambda_{0} I d\right)=1$, dim $\operatorname{ker}\left(S-\lambda_{1} I d\right)=n-1$ on $U=\left\{x: \lambda_{0}(x) \neq \lambda_{1}(x)\right\}$,

(d) $\lambda_{1}-\lambda_{0}=\frac{n-2}{4}\|\xi\|^{2}$ where $\xi=\omega^{\sharp} \in \mathfrak{i s o}(M)$.

In the addition $\lambda_{0}=\frac{1}{n}$ Scal ${ }_{g}^{D}$ where Scal ${ }_{g}^{D}=\operatorname{tr}_{g} \rho^{D}$ denotes the conformal scalar curvature of $(M, g, D)$.

Proof Note that $\omega(X)=g(\xi, X)$ where $\xi \in \mathfrak{i s o}(M)$ and the formula

$$
\rho^{\nabla}+\frac{1}{4}(n-2) \omega \otimes \omega=\Lambda g
$$

holds. Thus, $\nabla_{X}(\omega \otimes \omega)(X, X)=0$. From (1.4) it follows that

$$
\left(\nabla_{X} \rho\right)(X, X)=(X \Lambda) g(X, X) .
$$

It means that $(M, g) \in \mathcal{A} \oplus \mathcal{C}^{\perp}$ and $d\left(\Lambda-\frac{2}{n+2} \tau\right)=0$ ([9], Lemma 1.5), where $\tau$ is the scalar curvature of $(M, g)$. From (1.5) it follows that the tensor $T=S-\Lambda I d$ is a Killing tensor. Note that $\rho(\xi, \xi)=\left(\Lambda-\frac{1}{4}(n-2)\|\xi\|^{2}\right)\|\xi\|^{2}$ and if $X \perp \xi$ then $S X=\Lambda X$. Hence, the tensor $S$ has two eigenfunctions $\lambda_{0}=\Lambda-\frac{1}{4}(n-2)\|\xi\|^{2}$ and $\lambda_{1}=\Lambda$. This proves (b). Note that

$$
\tau=\lambda_{0}+(n-1) \lambda_{1}=n \Lambda-\frac{1}{4}(n-2)\|\xi\|^{2} .
$$

and $2 \tau-(n+2) \Lambda=C_{0}=$ const. Thus, $C_{0}=(n-2) \Lambda-\frac{1}{2}(n-2)\|\xi\|^{2}$. However, $(n-4) \lambda_{1}+2 \lambda_{0}=(n-2) \Lambda-\frac{1}{2}(n-2)\|\xi\|^{2}$ which proves (a). Note also that

$$
\frac{1}{n} s_{g}^{D}=\Lambda-\frac{n-2}{4}\|\xi\|^{2}=\lambda_{0}
$$

which finishes the proof. 
In the next theorem we show that conversely every Gray manifold satisfying the above conditions is in fact E-W manifold (see [9]).

Theorem 1.4 Let $(M, g)$ be a compact $\mathcal{A} \oplus \mathcal{C}^{\perp}$ manifold. Let us assume that the Ricci tensor $\rho$ of $(M, g)$ has exactly two eigenfunctions $\lambda_{0}, \lambda_{1}$ satisfying the conditions:

(a) $(n-4) \lambda_{1}+2 \lambda_{0}=C_{0}=$ const

(b) $\lambda_{0} \leq \lambda_{1}$ on $M$

(c) $\operatorname{dim} \operatorname{ker}\left(S-\lambda_{0} I d\right)=1, \operatorname{dim} \operatorname{ker}\left(S-\lambda_{1} I d\right)=n-1$ on $U=\left\{x: \lambda_{0}(x) \neq \lambda_{1}(x)\right\}$.

Then there exists a twofold Riemannian covering $\left(M^{\prime}, g^{\prime}\right)$ of $(M, g)$ and a Killing vector field $\xi \in \operatorname{iso}\left(M^{\prime}\right)$ such that $\left(M^{\prime},\left[g^{\prime}\right]\right)$ admits two different $E$-W structures with the standard metric $g^{\prime}$ and the corresponding 1 -forms $\omega_{\mp}=\mp \xi^{b}$ dual to the vector fields $\mp \xi$. In addition, $\lambda_{1}-\lambda_{0}=\frac{n-2}{4}\|\xi\|^{2}$. The condition (b) may be replaced by the condition

(b1) there exists a point $x_{0} \in M$ such that $\lambda_{0}\left(x_{0}\right)<\lambda_{1}\left(x_{0}\right)$.

Proof Note that $\tau=(n-1) \lambda_{1}+\lambda_{0}$ and $C_{0}=(n-4) \lambda_{1}+2 \lambda_{0}$. It follows that

$$
\lambda_{1}=\frac{2 \tau-C_{0}}{n+2}, \quad \lambda_{0}=\frac{(n-1) C_{0}-(n-4) \tau}{n+2} .
$$

In particular $\lambda_{0}, \lambda_{1} \in C^{\infty}(M)$. Let $S$ be the Ricci endomorphism of $(M, g)$ and let us define the tensor $T:=S-\lambda_{1} I d$. Since from (1.7) we have $d \lambda_{1}=\frac{2}{n+2} d \tau$ it follows that $T$ is a Killing tensor with two eigenfunctions: $\mu=0$ and $\lambda=\lambda_{0}-\lambda_{1}$. Hence there exists a twofold Riemannian covering $p:\left(M^{\prime}, g^{\prime}\right) \rightarrow(M, g)$ and a Killing vector field $\xi \in \mathfrak{i s o}\left(M^{\prime}\right)$ (see [9], th. 2.10) such that $S^{\prime} \xi=\left(\lambda_{0} \circ p\right) \xi$ where $S^{\prime}$ is the Ricci endomorphism of $\left(M^{\prime}, g^{\prime}\right)$. Note also that $\|\xi\|^{2}=|\lambda-\mu|=\left|\lambda_{0}-\lambda_{1}\right|$. Let us define the 1 -form $\omega$ on $M^{\prime}$ by $\omega=c \xi^{b}$ where $c=2 \sqrt{\frac{1}{n-2}}$. It is easy to check that with such a choice of $\omega$ Eq. (1.4) is satisfied and $\delta \omega=0$. Thus $\left(M^{\prime}, g^{\prime}, \omega\right)$ defines an E-W structure and $g^{\prime}$ is the standard metric for $\left(M^{\prime},\left[g^{\prime}\right]\right)$. Note that $\left(M, g^{\prime},-\omega\right)$ gives another E-W structure corresponding to the field $-\xi$.

Corollary 1.5 Let $(M, g)$ be a compact simply connected manifold satisfying the assumptions of Th. 1.4. Then $(M,[g])$ admits two E-W structures with the standard metric $g$.

\section{Killing tensors}

In this section, we describe the Riemannian manifold $(M, g)$ where $g \in[g]$ is the standard metric of E-W structure $(M,[g], D)$.

We say, that a distribution (not necessarily integrable) $\mathcal{D}$ is totally geodesic, if $\nabla_{X} X \in$ $\Gamma(\mathcal{D})$ for every $X \in \Gamma(\mathcal{D})$. Note that if $(M, g)$ is a compact E-W manifold with the Gauduchon metric then the distribution $\mathcal{D}_{\lambda_{1}}$ is totally geodesic since is orthogonal to the distribution spanned by a Killing vector field.

We start with:

Lemma 2.1 Let $S$ be a self-adjoint tensor on $(M, g)$ with exactly two eigenvalues $\lambda, \mu$. If the distributions $\mathcal{D}_{\lambda}, \mathcal{D}_{\mu}$ are both umbilical, $\nabla \lambda \in \Gamma\left(D_{\mu}\right), \nabla \mu \in \Gamma\left(D_{\lambda}\right)$ and the mean curvature normals $\xi_{\lambda}, \xi_{\mu}$ of the distributions $D_{\lambda}, D_{\mu}$ respectively satisfy the equations

$$
\xi_{\lambda}=\frac{1}{2(\mu-\lambda)} \nabla \lambda, \quad \xi_{\mu}=\frac{1}{2(\lambda-\mu)} \nabla \mu
$$

then $S$ is a Killing tensor. 
Proof We have to show that $g(\nabla S(Z, Z), Z)=0$ for arbitrary $Z \in T M$. Let $Z=X+Y$ where $X \in \mathcal{D}_{\lambda}, Y \in \mathcal{D}_{\mu}$. Then

$$
\begin{aligned}
g(\nabla S(Z, Z), Z)= & g(\nabla S(X, X), X)+2 g(\nabla S(X, X), Y)+g(\nabla S(Y, X), X) \\
& +2 g(\nabla S(Y, Y), X)+g(\nabla S(X, Y), Y)+g(\nabla S(Y, Y), Y) .
\end{aligned}
$$

Since $\nabla S(X, X)=(\lambda-\mu) g(X, X) \xi_{\lambda}, \nabla S(Y, Y)=(\mu-\lambda) g(Y, Y) \xi_{\mu}$ and

$$
g(\nabla S(X, X), X)=0, g(\nabla S(Y, Y), Y)=0
$$

one can easily check that $g(\nabla S(Z, Z), Z)=0$.

Remark We call here a vector field $\xi$ the mean curvature normal of a umibilical distribution $\mathcal{D}$ if for every $X \in \Gamma(\mathcal{D})$ we have $\pi\left(\nabla_{X} X\right)=g(X, X) \xi$ where $\pi$ is a projection on the orthogonal complement of $\mathcal{D}$. Note that $\mathcal{D}$ may not be integrable.

Proposition 2.2 Let $(M, g)$ be a $2 n$-D Riemannian manifold whose Ricci tensor $\rho$ has two eigenvalues $\lambda_{0}(x), \lambda_{1}(x)$ of multiplicity 1 and $2 n-1$, respectively, at every point $x$ of $M$. Assume that the eigendistribution $\mathcal{D}_{\lambda_{1}}$ corresponding to $\lambda_{1}$ is totally geodesic. Then $(M, g)$ is a Gray manifold if and only if $2 \lambda_{0}+(2 n-4) \lambda_{1}$ is constant and $\nabla \tau \in \Gamma\left(\mathcal{D}_{\lambda_{1}}\right)$.

Proof Let $S_{0}$ be the Ricci endomorphism of $(M, g)$, i.e., $\rho(X, Y)=g\left(S_{0} X, Y\right)$. Let $S$ be the tensor defined by the formula

$$
S_{0}=S+\frac{\tau}{n+1} \text { id }
$$

Then

$$
\operatorname{tr} S=-\frac{(n-1) \tau}{n+1}
$$

Let $\lambda_{0}, \lambda_{1}$ be the eigenfunctions of $S_{0}$ and let us assume that

$$
2 \lambda_{0}+(2 n-4) \lambda_{1}=C
$$

where $C \in \mathbb{R}$. Note that $S$ also has two eigenfunctions which we denote by $\lambda_{0}^{\prime}, \lambda_{1}^{\prime}$, respectively. It is easy to see that $\lambda_{0}^{\prime}=-\frac{n-1}{n+1} \tau+C \frac{2 n-1}{2(n+1)}, \lambda_{1}^{\prime}=-\frac{C}{2(n+1)}$ and $\lambda_{0}=-\frac{\tau(n-2)}{n+1}+$ $C \frac{2 n-1}{2(n+1)}, \lambda_{1}=\frac{\tau}{(n+1)}-\frac{C}{2(n+1)}$. Since the distribution $\mathcal{D}_{\lambda_{0}}$ is umbilical we have $\nabla_{X} X_{\mid \mathcal{D}_{\lambda_{1}}}=$ $g(X, X) \xi$ for any $X \in \Gamma\left(\mathcal{D}_{\lambda_{0}}\right)$ where $\xi$ is the mean curvature normal of $\mathcal{D}_{\lambda_{0}}$. Since the distribution $\mathcal{D}_{\lambda_{1}}$ is totally geodesic we also have $\nabla_{X} X_{\mid \mathcal{D}_{\lambda_{0}}}=0$ for any $X \in \Gamma\left(\mathcal{D}_{\lambda_{1}}\right)$. Let $\left\{E_{1}, E_{2}, E_{3}, E_{4}, \ldots, E_{2 n-1}, E_{2 n}\right\}$ be a local orthonormal basis of $T M$ such that $\mathcal{D}_{\lambda_{0}}=$ span $\left\{E_{1}\right\}$ and $\mathcal{D}_{\lambda_{1}}=\operatorname{span}\left\{E_{2}, E_{3}, E_{4}, \ldots, E_{2 n}\right\}$. Then $\nabla_{E_{i}} E_{i \mid \mathcal{D}_{\lambda_{0}}}=0$ for $i \in\{2,3,4, \ldots, 2 n\}$ and

$$
\nabla_{E_{1}} E_{1 \mid \mathcal{D}_{\lambda_{1}}}=\xi
$$

Consequently (note that $\nabla \lambda_{0 \mid \mathcal{D}_{\lambda_{0}}}^{\prime}=0$ if and only if $\nabla \tau_{\mid \mathcal{D}_{\lambda_{0}}}=0$ ),

$$
\begin{aligned}
\operatorname{tr}_{g} \nabla S & =\sum_{i=1}^{n} \nabla S\left(E_{i}, E_{i}\right)=-\left(S-\lambda_{0} \mathrm{id}\right)\left(\nabla_{E_{1}} E_{1}\right)+\nabla \lambda_{0 \mid \mathcal{D}_{\lambda_{0}}} \\
& =-\left(\lambda_{1}-\lambda_{0}\right) \xi
\end{aligned}
$$


if we assume that $\nabla \tau_{\mid \mathcal{D}_{\lambda_{0}}}=0$. On the other hand, $\operatorname{tr}_{g} \nabla S_{0}=\frac{\nabla \tau}{2}$ and $\operatorname{tr}_{g} \nabla S=\operatorname{tr}_{g} \nabla S_{0}-\frac{\nabla \tau}{n+1}$. Consequently,

$$
\operatorname{tr}_{g} \nabla S=\frac{(n-1) \nabla \tau}{2(n+1)}=-\frac{1}{2} \nabla \lambda_{0}^{\prime} .
$$

Thus, $\xi=-\frac{1}{2\left(\lambda_{0}-\lambda_{1}\right)} \nabla \lambda_{0}^{\prime}$. From the Lemma it follows that $(M, g)$ is an $\mathcal{A} \oplus \mathcal{C}^{\perp}$-manifold if $2 \lambda_{0}+(n-4) \lambda_{1}$ is constant and $\nabla \tau \in \Gamma\left(\mathcal{D}_{\lambda_{1}}\right)$. These conditions are also necessary since $\nabla \lambda_{1}^{\prime}=0$ if $(M, g)$ is an $\mathcal{A} \oplus \mathcal{C}^{\perp}$-manifold and $\mathcal{D}_{\lambda_{1}}$ is totally geodesic. Analogously $\xi=-\frac{1}{2\left(\lambda_{0}^{\prime}-\lambda_{1}^{\prime}\right)} \nabla \lambda_{0}^{\prime}$ and $\nabla \lambda_{0}^{\prime}=-\frac{n-1}{(n+1)} \nabla \tau \in \Gamma\left(\mathcal{D}_{\lambda_{1}}\right)$, where $\xi$ is the mean curvature normal of the umbilical distribution $\mathcal{D}_{\lambda_{0}}$, if $(M, g)$ is an $\mathcal{A} \oplus \mathcal{C}^{\perp}$-manifold.

\section{Conformally Kähler E-W manifolds}

Let $g$ be the standard metric of $(M,[g])$. Now let us recall that $\rho^{D}(X, Y)=\lambda_{0} g(X, Y)+$ $\frac{n}{4} d \omega(X, Y)$. Let us assume that $(M, J)$ is complex and $[g]$ is Hermitian i.e. $g(J X, J Y)=$ $g(X, Y)$. It follows that $\rho^{D}$ is $J$-invariant if and only if $d \omega$ is a $(1,1)$ form, $d \omega(J X, J Y)=$ $d \omega(X, Y)$. Since $\omega(X)=g(\xi, X)$ it follows that $d \omega$ is a $(1,1)$ form iff $\nabla_{J X} \xi=J \nabla_{X} \xi$.

Proposition 3.1 Let $(M, J)$ be a compact complex manifold with conformal Hermitian structure $[g]$. Let us assume that $[g]$ is conformally Kähler and $f^{2} g$ is a Kähler metric on $(M, J)$ where $g$ is the standard metric and $f \in C^{\infty}(M)$. If $(M,[g])$ is $E$-W with J-invariant Ricci tensor $\rho^{D}$ then $J \xi$ is colinear with $\nabla f$ in $U=\left\{x: \xi_{x} \neq 0\right\}$ and $\xi$ is a holomorphic Killing field on $\left(M, f^{2} g, J\right)$.

Proof Let $\nabla$ be a Levi-Civita connection of the standard metric $g$ and $\nabla^{1}$ be a Levi-Civita connection of the Kähler metric $g_{1}=f^{2} g$. Note that $\xi$ is a conformal field on $\left(M, g_{1}\right)$, $L_{\xi} g_{1}=L_{\xi}\left(f^{2} g\right)=2 \xi \ln f g_{1}=\sigma g_{1}$. Every conformal field on a compact Kähler manifold is Killing (see [12]), hence consequently $\xi f=0$ and $\xi \in \mathfrak{i s o}\left(M, g_{1}\right)$. On a Kähler compact manifold every Killing vector field is holomorphic (see [14]). Thus, $\xi \in \mathfrak{h o l}(M, J)$. Note that

$$
\nabla_{X} \xi=\nabla_{X}^{1} \xi-\mathrm{d} \ln f(X) \xi-\mathrm{d} \ln f(\xi) X+g_{1}(X, \xi) \nabla^{1} \ln f
$$

Thus,

$$
\begin{aligned}
\nabla_{J X} \xi-J \nabla_{X} \xi= & -\mathrm{d} \ln f(J X) \xi-\mathrm{d} \ln f(\xi) J X+g_{1}(J X, \xi) \nabla^{1} \ln f \\
& +\mathrm{d} \ln f(X) J \xi+\mathrm{d} \ln f(\xi) J X-g_{1}(X, \xi) J \nabla^{1} \ln f .
\end{aligned}
$$

Hence, $\nabla_{J X} \xi=J \nabla_{X} \xi$ if

$$
-\mathrm{d} \ln f(J X) \xi+g_{1}(J X, \xi) \nabla^{1} \ln f+\mathrm{d} \ln f(X) J \xi-g_{1}(X, \xi) J \nabla^{1} \ln f=0 .
$$

Put $X=\xi$ then we get $g_{1}(\xi, \xi) J \nabla^{1} \ln f=-\mathrm{d} \ln f(J \xi) \xi$. It follows that in $U=\{x \in M$ : $\left.\xi_{x} \neq 0\right\}$ there exists a smooth function $\phi$ such that $\nabla^{1} f=\phi J \xi$.

Let us recall the definition of a special Kähler-Ricci potential ([5,3]).

Definition A nonconstant function $\tau \in C^{\infty}(M)$, where $(M, g, J)$ is a Kähler manifold, is called a special Kähler-Ricci potential if the field $X=J(\nabla \tau)$ is a Killing vector field and at every point with $d \tau \neq 0$ all nonzero tangent vectors orthogonal to the fields $X, J X$ are eigenvectors of both $\nabla d \tau$ and the Ricci tensor $\rho$ of $(M, g, J)$. 
Now our aim is to prove

Theorem 3.2 Let us assume that $(M,[g], J)$ is a compact, conformally Kähler E-W manifold with Hermitian Ricci tensor $\rho^{D}$ which is not conformally Einstein. Then the conformally equivalent Kähler manifold $\left(M, g_{1}, J\right)$ admits a holomorphic Killing field with a KählerRicci potential. Thus, $M=\mathbb{P}(L \oplus \mathcal{O})$ where $L$ is a holomorphic line bundle over a compact Kähler Einstein manifold $(N, h)$ of positive scalar curvature or is a complex projective space $\mathbb{C} \mathbb{P}^{n}$.

Proof Let $\rho, \rho^{1}$ be the Ricci tensors of conformally related riemannian metrics $g, g_{1}=f^{2} g$. Then

$$
\rho=\rho^{1}+(n-2) f^{-1} \nabla^{1} d f+\left[f^{-1} \Delta^{1} f-(n-1) f^{-2} g_{1}\left(\nabla^{1} f, \nabla^{1} f\right)\right] g_{1} .
$$

Note that for arbitrary $X, Y \in \mathfrak{X}(M)$ we have $\nabla^{1} d f(X, Y)=g_{1}\left(\nabla_{X}^{1} \nabla^{1} f, Y\right)=g_{1}$ $(X \phi J \xi, Y)+\phi g_{1}\left(J \nabla_{X}^{1} \xi, Y\right)$. Thus for any $X, Y \in \mathfrak{X}(M)$ :

$$
\begin{aligned}
\rho(X, Y)-(n-2) f X \phi g(J \xi, Y)= & \rho^{1}(X, Y)+(n-2) f^{-1} \phi g_{1}\left(J \nabla_{X}^{1} \xi, Y\right) \\
+ & {\left[f^{-1} \Delta^{1} f-(n-1) f^{-2} g_{1}\left(\nabla^{1} f, \nabla^{1} f\right)\right] } \\
& g_{1}(X, Y),
\end{aligned}
$$

where $\Delta^{1} f=\operatorname{tr}_{g_{1}} \nabla^{1} d f$.

We shall show that $\xi$ has zeros on $M$. If $\xi \neq 0$ on $M$ then the function $\phi$ would be defined and smooth on the whole of $M$. Since $M$ is compact it would imply that there exists a point $x_{0} \in M$ such that $d \phi=0$ at $x_{0}$. On the other hand, the eigenvalues $\lambda_{0}, \lambda_{1}$ of the Ricci tensor $\rho$ satisfy $\lambda_{0}-\lambda_{1}=C g(\xi, \xi)$ where $C \neq 0$ is a real number. Since $\xi \neq 0$ it follows that the eigenvalues of $\rho$ do not coincide at any point of $M$. In particular $\rho$ is not $J$-invariant at $x_{0}$, a contradiction, since the right hand part of (3.1) is $J$-invariant. It implies that $\xi$ is a holomorphic Killing vector field with zeros and thus has a potential $\tau$ (see [11]), i.e., there exists $\tau \in C^{\infty}(M)$ such that $\xi=J \nabla^{1} \tau$. Hence, $\mathrm{d} f=-\phi \mathrm{d} \tau$ and $\mathrm{d} \phi \wedge \mathrm{d} \tau=0$. It implies that $\mathrm{d} \phi=\alpha \mathrm{d} \tau$. Thus, we have for arbitrary $X, Y \in \mathfrak{X}(M)$ :

$$
\begin{aligned}
& \rho(X, Y)+(n-2) f^{-1} \alpha \mathrm{d} \tau(X) \mathrm{d} \tau(Y)=\rho^{1}(X, Y) \\
& \quad-(n-2) f^{-1} \phi H^{\tau}(X, Y)-\left[f^{-1} \alpha Q+f^{-1} \phi \Delta^{1} \tau+(n-1) f^{-2} \phi^{2} Q\right] g_{1}(X, Y) .
\end{aligned}
$$

where $Q=g_{1}(\xi, \xi)$.

Note that the tensor $\widetilde{\rho}(X, Y)=\rho(X, Y)+(n-2) f^{-1} \alpha \mathrm{d} \tau(X) \mathrm{d} \tau(Y)$ is $J$-invariant. In particular $\widetilde{\rho}(\xi, \xi)=\lambda_{0} g(\xi, \xi)=\lambda_{o} \frac{Q}{f^{2}}$. On the other hand, $\widetilde{\rho}\left(\nabla^{1} \tau, \nabla^{1} \tau\right)=\lambda_{1} \frac{Q}{f^{2}}+(n-$ 2) $f^{-1} \alpha Q^{2}$. Hence, $\left(\lambda_{0}-\lambda_{1}\right) \frac{Q}{f^{2}}=(n-2) f^{-1} \alpha Q^{2}$. Since $\lambda_{0}-\lambda_{1}=-\frac{1}{4}(n-2) \frac{Q}{f^{2}}$ we get $\alpha=-\frac{1}{4 f^{3}}$. Hence

$$
\mathrm{d} \phi=-\frac{1}{4 f^{3}} \mathrm{~d} \tau=\frac{1}{4 f^{3}} \frac{d f}{\phi},
$$

and we get $8 \phi \mathrm{d} \phi=-\mathrm{d}\left(\frac{1}{f^{2}}\right)$. Hence, $\mathrm{d}\left(4 \phi^{2}+\frac{1}{f^{2}}\right)=0$ and $4 \phi^{2}+\frac{1}{f^{2}}=C=$ const.

Let us denote $\chi=(n-2) f^{-1} \phi, \sigma_{0}=f^{-1} \alpha Q+f^{-1} \phi \Delta^{1} \tau+(n-1) f^{-2} \phi^{2} Q$. Note also that the vector field $v=\nabla^{1} \tau$ is holomorphic and consequently $i_{v} \rho^{1}=-\frac{1}{2} \mathrm{~d} \Delta^{1} \tau=-\frac{1}{2} \mathrm{~d} Z$ where $Z=\Delta^{1} \tau$. From the equation

$$
\begin{aligned}
\widetilde{\rho}(X, Y)= & \rho^{1}(X, Y)-(n-2) f^{-1} \phi H^{\tau}(X, Y)- \\
& {\left[f^{-1} \alpha Q+f^{-1} \phi \Delta^{1} \tau+(n-1) f^{-2} \phi^{2} Q\right] g_{1}(X, Y) . }
\end{aligned}
$$


valid for arbitrary $X, Y \in \mathfrak{X}(M)$ we get

$$
\frac{\lambda_{0}}{f^{2}} \mathrm{~d} \tau=-\frac{1}{2} \mathrm{~d} Z+\frac{1}{2} \chi \mathrm{d} Q-\sigma_{0} \mathrm{~d} \tau,
$$

and

$$
\mathrm{d} Z=\chi \mathrm{d} Q-2 \sigma \mathrm{d} \tau
$$

where $\sigma=\sigma_{0}+\frac{\lambda_{0}}{f^{2}}$. From (3.5) we obtain

$$
\mathrm{d} \chi \wedge \mathrm{d} Q-2 \mathrm{~d} \sigma \wedge \mathrm{d} \tau=0
$$

Since $\mathrm{d} \chi=\gamma \mathrm{d} \tau$ we have $\mathrm{d} \tau \wedge(\gamma \mathrm{d} Q+2 \mathrm{~d} \sigma)=0$ which implies $\gamma \mathrm{d} Q+2 \mathrm{~d} \sigma=\kappa \mathrm{d} \tau$ for a certain function $\kappa$. Note that

$$
\begin{array}{r}
\mathrm{d} \sigma_{0}=f^{-1} \alpha \mathrm{d} Q+f^{-1} \phi \mathrm{d} Z+(n-1) f^{-2} \phi^{2} \mathrm{~d} Q+h \mathrm{~d} \tau= \\
{\left[-\frac{1}{4 f^{4}}+(n-1) f^{-2} \phi^{2}\right] \mathrm{d} Q+f^{-1} \phi \mathrm{d} Z+h \mathrm{~d} \tau,}
\end{array}
$$

for a certain function $h$. On the other hand, $\lambda_{0}=-\frac{(n-4) Q}{4 f^{2}}+\frac{C_{0}}{n-2}$. Hence, $\mathrm{d}\left(\frac{\lambda_{0}}{f^{2}}\right)=$ $-\frac{(n-4)}{4 f^{4}} \mathrm{~d} Q+k \mathrm{~d} \tau$ and

$$
\mathrm{d} \sigma=\left[-\frac{1}{4 f^{4}}+(n-1) f^{-2} \phi^{2}-\frac{(n-4)}{4 f^{4}}\right] \mathrm{d} Q+f^{-1} \phi \mathrm{d} Z+l \mathrm{~d} \tau
$$

for some functions $k, l$. Since $\mathrm{d} \chi=(n-2) \mathrm{d}\left(f^{-1} \phi\right)=-\frac{(n-2)}{4 f^{4}}\left(1-4 \phi^{2} f^{2}\right) \mathrm{d} \tau$ we have $\gamma=-\frac{(n-2)}{4 f^{4}}\left(1-4 \phi^{2} f^{2}\right)$ and

$$
\left[-\frac{3 n-8}{4 f^{4}}+\frac{(3 n-4) \phi^{2}}{4 f^{2}}\right] \mathrm{d} Q+2 f^{-1} \phi \mathrm{d} Z=m \mathrm{~d} \tau,
$$

for a certain function $m$.

From Eqs. (3.5) and (3.7) it follows that

$$
\mathrm{d} Q \wedge \mathrm{d} \tau=\mathrm{d} Z \wedge \mathrm{d} \tau=0
$$

on a dense subset of $M$ and hence everywhere.

Define a distribution $\mathcal{D}=\operatorname{span}\{\xi, J \xi\}$ and let $\mathcal{D}^{\perp}$ be an orthogonal (with respect to $g$ so also with respect to $g_{1}$ ) complement to $\mathcal{D}$. Both distributions are defined in an open dense subset $U=\left\{x: \xi_{x} \neq 0\right\}$. Let $\pi_{\mathcal{D}}, \pi_{\mathcal{D}^{\perp}}$ be orthogonal projections on $\mathcal{D}, \mathcal{D}^{\perp}$, respectively. Let us define $\omega_{\mathcal{D}}(X, Y)=g_{1}\left(J \pi_{\mathcal{D}} X, Y\right), \omega_{\mathcal{D}^{\perp}}(X, Y)=g_{1}\left(J \pi_{\mathcal{D}^{\perp}} X, Y\right)$. Then $\omega_{\mathcal{D}}+\omega_{\mathcal{D}^{\perp}}=\Omega$ where $\Omega(X, Y)=g_{1}(J X, Y)$ is the Kähler form of $\left(M, g_{1}, J\right)$. Note that $\omega_{\mathcal{D}}=\frac{1}{Q} d \tau \wedge$ $d^{c} \tau$. Since $\xi$ is a holomorphic Killing field on $\left(M, g_{1}, J\right)$ it follows that $H^{\tau}(J X, Y)=$ $\frac{1}{2} d d^{c} \tau(X, Y)$. Since $\nabla_{v}^{1} v=-\frac{1}{2} \nabla^{1} Q=c v$ it follows that $\mathcal{D}$ is an eigendistribution of both $\rho^{1}$ and $d d^{c} \tau$. We have (we denote the Ricci form also by $\rho^{1}$ )

$$
\begin{aligned}
\rho^{1} & =\lambda \omega_{\mathcal{D}}+\omega_{1} \\
\frac{1}{2} \mathrm{dd}^{c} \tau & =\mu \omega_{\mathcal{D}}+\omega_{2},
\end{aligned}
$$

where $\lambda, \mu$ are eigenvalues of $\rho^{1}, H^{\tau}$ corresponding to an eigen distribution $\mathcal{D}$. The eigenvalue $\mu$ satisfies an equation $\mu Q=H^{\tau}\left(\nabla^{1} \tau, \nabla^{1} \tau\right)=-\frac{1}{2} \mathrm{~d} Q\left(\nabla^{1} \tau\right)=-\frac{1}{2} \beta Q$ where 
$\mathrm{d} Q=\beta d \tau$. Hence, $\mu=-\frac{1}{2} \beta$ and $\mathrm{d} \mu \wedge \mathrm{d} \tau=0$. From (3.5) it is clear that also $\mathrm{d} \lambda \wedge \mathrm{d} \tau=0$.

Now we have

$$
\widetilde{\rho}=\rho^{1}-\frac{1}{2} \chi \operatorname{dd}^{c} \tau-\sigma_{0} \Omega
$$

and consequently

$$
\frac{\lambda_{0}}{f^{2}} \omega_{\mathcal{D}}=\lambda \omega_{\mathcal{D}}-\chi \mu \omega_{\mathcal{D}}-\sigma_{0} \omega_{\mathcal{D}}
$$

and

$$
\frac{\lambda_{1}}{f^{2}} \omega_{\mathcal{D}^{\perp}}=\omega_{1}-\chi \omega_{2}-\sigma_{0} \omega_{\mathcal{D}^{\perp}}
$$

From (3.11) we obtain $\lambda-\mu \chi=\sigma_{0}+\frac{\lambda_{0}}{f^{2}}$. Hence,

$$
\omega_{1}-\chi \omega_{2}=\left(\sigma_{0}+\frac{\lambda_{1}}{f^{2}}\right) \omega_{\mathcal{D}^{\perp}}=\left(\lambda-\mu \chi+\frac{\lambda_{1}-\lambda_{0}}{f^{2}}\right) \omega_{\mathcal{D}^{\perp}}=\sigma_{1} \omega_{\mathcal{D}^{\perp}} .
$$

From (3.9) we get

$$
\lambda \mathrm{d} \omega_{\mathcal{D}}=-\mathrm{d} \omega_{1}, \mu \mathrm{d} \omega_{\mathcal{D}}=-\mathrm{d} \omega_{2} .
$$

Equation (3.13) implies that

$$
\mathrm{d} \omega_{1}-\mathrm{d} \chi \wedge \omega_{2}-\chi \mathrm{d} \omega_{2}=\mathrm{d} \sigma_{1} \wedge \omega_{\mathcal{D}^{\perp}}+\sigma_{1} \mathrm{~d} \omega_{\mathcal{D}^{\perp}}
$$

thus

$$
\left(-\lambda+\mu \chi+\sigma_{1}\right) \mathrm{d} \omega_{\mathcal{D}}=\mathrm{d} \chi \wedge \omega_{2}+\mathrm{d} \sigma_{1} \wedge \omega_{\mathcal{D}^{\perp}} .
$$

Note that $\mathrm{d} \omega_{\mathcal{D}}=\mathrm{d}\left(\frac{1}{Q} \mathrm{~d} \tau \wedge \mathrm{d}^{c} \tau\right)=-\frac{1}{Q} \mathrm{~d} \tau \wedge \mathrm{dd}^{c} \tau=-\frac{2}{Q} \mathrm{~d} \tau \wedge\left(\mu \omega_{\mathcal{D}}+\omega_{2}\right)=-\frac{2}{Q} \mathrm{~d} \tau \wedge \omega_{2}$ and $\lambda_{1}-\lambda_{0}=\frac{1}{4}(n-2) \frac{Q}{f^{2}}$. Let us write $d \sigma_{1}=\psi \mathrm{d} \tau$, then we obtain

$$
\mathrm{d} \tau \wedge\left(\frac{n-2}{4 f^{4}}\left(-1-4 f^{2} \phi^{2}\right) \omega_{2}-\psi \omega_{\mathcal{D}^{\perp}}\right)=0 .
$$

From (3.14) it is clear that in $U$ we have $\omega_{2}=\kappa_{2} \omega_{\mathcal{D}^{\perp}}$ for a certain function $\kappa_{2} \in C^{\infty}(U)$. Hence, also $\omega_{1}=\kappa_{1} \omega_{\mathcal{D}^{\perp}}$ for a certain function $\kappa_{1} \in C^{\infty}(U)$. It follows that the function $\tau$ is a Kähler-Ricci potential. The fact that the Einstein-Kähler manifold $(N, h)$ has a positive scalar curvature is proved below. It is easy to check that also for $\operatorname{dim} M=4$ the manifold $(N, h)$ has constant scalar curvature. The E-W structure on these manifolds is described in [13], [18].

\section{Eigenvalues of the Ricci tensor}

In our construction we shall follow Bérard Bergery (see [1,10]). Let $(N, h, J)$ be a compact Kähler Einstein manifold and $\operatorname{dim} N=2 m, s \geq 0, L>0, s \in \mathbb{Q}, L \in \mathbb{R}$, and $g:[0, L] \rightarrow \mathbb{R}$ be a positive, smooth function on $[0, L]$ which is even at 0 and $L$, i.e., there exists an $\epsilon>0$ and even, smooth functions $g_{1}, g_{2}:(-\epsilon, \epsilon) \rightarrow \mathbb{R}$ such that $g(t)=g_{1}(t)$ for $t \in[0, \epsilon)$ and $g(t)=g_{2}(L-t)$ for $t \in(L-\epsilon, L]$. Let $f:(0, L) \rightarrow \mathbb{R}$ be positive on $(0, L), f(0)=f(L)=0$ and let $f$ be odd at the points $0, L$. Let $P$ be a circle bundle over $N$ classified by the integral cohomology class $\frac{s}{2} c_{1}(N) \in H^{2}(N, \mathbb{R})$ if $c_{1}(N) \neq 0$. Let $q$ be 
the unique positive integer such that $c_{1}(N)=q \alpha$ where $\alpha \in H^{2}(N, \mathbb{R})$ is an indivisible integral class. Such a $q$ exists if $N$ is simply connected or $\operatorname{dim} N=2$. Note that every Kähler Einstein manifold with positive scalar curvature is simply connected. Then

$$
s=\frac{2 k}{q} ; k \in \mathbb{Z} .
$$

It is known that $q=n$ if $N=\mathbb{C P}^{n-1}$ (see [2], p. 273). Note that $c_{1}(N)=\left\{\frac{1}{2 \pi} \rho_{N}\right\}=$ $\left\{\frac{\tau_{N}}{4 m \pi} \omega_{N}\right\}$ where $\rho_{N}=\frac{\tau_{N}}{2 m} \omega_{N}$ is the Ricci form of $(N, h, J), \tau_{N}$ is the scalar curvature of $(N, h)$ and $\omega_{N}$ is the Kähler form of $(N, h, J)$. We can assume that $\tau_{N}= \pm 4 m$. In the case $c_{1}(N)=0$ we shall assume that $(N, h, J)$ is a Hodge manifold, i.e., the cohomology class $\left\{\frac{s}{2 \pi} \omega_{N}\right\}$ is an integral class. On the bundle $p: P \rightarrow N$ there exists a connection form $\theta$ such that $\mathrm{d} \theta=s p^{*} \omega_{N}$ where $p: P \rightarrow N$ is the bundle projection. Let us consider the manifold $U_{s, f, g}=(0, L) \times P$ with the metric

$$
k=\mathrm{d} t^{2}+f(t)^{2} \theta^{2}+g(t)^{2} p^{*} h .
$$

It is known that the metric (4.1) extends to a metric on the sphere bundle $M=P \times{ }_{S^{1}} \mathbb{C P}^{1}$ if and only if a function $g$ is positive and smooth on $[0, L]$, even at the points $0, L$, the function $f$ is positive on $(0, L)$, smooth and odd at $0, L$ and additionally

$$
f^{\prime}(0)=1, \quad f^{\prime}(L)=-1
$$

Then, the metric (4.1) is bi-Hermitian (see [10]). Note that $M=\mathbb{P}(L \oplus \mathcal{O})$ where $L=P \times{ }_{S^{1}} \mathbb{C}$ with $S^{1}$ acting in a standard way on $\mathbb{C}$ and $\mathcal{O}$ is the trivial line bundle over $N$.

The metric $k=k_{f, g}$ extends to a metric on $\mathbb{C P}^{n}$ if and only if the function $g$ is positive and smooth on $[0, L)$, even at 0 , odd at $L$, the function $f$ is positive, smooth and odd at $0, L$ and additionally

$$
f^{\prime}(0)=1, \quad f^{\prime}(L)=-1, \quad g(L)=0, \quad g^{\prime}(L)=-1 .
$$

Let us assume that $(N, h)$ is a $2(n-1)$-D Kähler-Einstein manifold of scalar curvature $4(n-1) \epsilon$ where $\epsilon \in\{-1,0,1\}$. Using the results in Sect. 3 and [10] we obtain the following formulae for the eigenvalues of the Ricci tensor $\rho$ of $\left(U_{s, f, g}, k_{f, g}\right)$ :

$$
\begin{aligned}
& \lambda_{0}=-2(n-1) \frac{g^{\prime \prime}}{g}-\frac{f^{\prime \prime}}{f}, \\
& \lambda_{1}=-\frac{f^{\prime \prime}}{f}+2(n-1)\left(\frac{s^{2} f^{2}}{4 g^{4}}-\frac{f^{\prime} g^{\prime}}{f g}\right), \\
& \lambda_{2}=-\frac{g^{\prime \prime}}{g}+\left(\frac{s^{2} f^{2}}{4 g^{4}}-\frac{f^{\prime} g^{\prime}}{f g}\right)+\frac{2 \epsilon}{g^{2}}-\frac{3 s^{2} f^{2}}{4 g^{4}}-(2 n-3) \frac{\left(g^{\prime}\right)^{2}}{g^{2}} .
\end{aligned}
$$

We shall show that in fact $\epsilon=1$, i.e., the scalar curvature of the Einstein manifold $(N, h, J)$ is positive. From [9], p. 17, th. 3.8. it follows that the conformal scalar curvature of E-W manifold and hence $\lambda_{1}$ is nonnegative. We also have for the Gauduchon metric $\lambda_{0}=\lambda_{2}$ and $\lambda_{1}+C^{2} f^{2}=\lambda_{0}$ for a positive constant $C$. Since $f(0)=0=f(L)$ it follows that $f$ attains a maximum at a point $t_{0} \in(0, L)$. Then $f^{\prime}\left(t_{0}\right)=0$ and $f^{\prime \prime}\left(t_{0}\right) \leq 0$. Hence at $t_{0}$ we have

$$
\lambda_{1}=-\frac{f^{\prime \prime}}{f}+2(n-1) \frac{s^{2} f^{2}}{4 g^{4}}>0
$$

and

$$
-\frac{g^{\prime \prime}}{g}+\frac{2 \epsilon}{g^{2}}-\frac{s^{2} f^{2}}{2 g^{4}}-(2 n-3) \frac{\left(g^{\prime}\right)^{2}}{g^{2}}=-2(n-1) \frac{g^{\prime \prime}}{g}-\frac{f^{\prime \prime}}{f}
$$


and hence

$$
\frac{2 \epsilon}{g^{2}}=\frac{s^{2} f^{2}}{2 g^{4}}+(2 n-3) \frac{\left(g^{\prime}\right)^{2}}{g^{2}}-(2 n-3) \frac{g^{\prime \prime}}{g}-\frac{f^{\prime \prime}}{f} .
$$

From (4.2) it follows that at $t_{0}$

$$
-2(n-1) \frac{g^{\prime \prime}}{g}=2(n-1) \frac{s^{2} f^{2}}{4 g^{4}}+C^{2} f^{2}>0
$$

and consequently $\epsilon>0$.

Acknowledgments The author thanks the referee for his valuable remarks, which improved the paper. The paper was supported by Narodowe Centrum Nauki grant no. DEC-2011/01/B/ST1/02643.

Open Access This article is distributed under the terms of the Creative Commons Attribution License which permits any use, distribution, and reproduction in any medium, provided the original author(s) and the source are credited.

\section{References}

1. Bérard Bergery, L.: Sur de nouvelles variétés riemanniennes d'Einstein. Publ. de l'Inst. E. Cartan (Nancy) 4, 1-60 (1982)

2. Besse, A.: Einstein manifolds. Springer, Berlin, HD (1987)

3. Derdziński, A., Maschler, G.: Local classification of conformally-Einstein Kähler metrics in higher dimension. Proc. London Math. Soc. (3) 87(3), 779-819 (2003)

4. Derdziński, A., Maschler, G.: A moduli curve for compact conformally-Einstein Kähler manifolds. Compos. Math. 141(4), 1029-1080 (2005)

5. Derdziński, A., Maschler, G.: Special Kähler-Ricci potentials on compact Kähler manifolds. J. Reine Angew. Math. 593, 73-116 (2006)

6. Dunajski, M., Tod, K.P.: Four dimensional metrics conformal to Kähler. In: Mathematical Proceedings of the Cambridge Philosophical Society, Cambridge (2010)

7. Gauduchon, P.: La 1-forme de torsion d'une variete hermitienne compacte. Math. Ann. 267, 495$518(1984)$

8. Gray, A.: Einstein-like manifolds which are not Einstein. Geom. Dedicata 7, 259-280 (1978)

9. Jelonek, W.: Killing tensors and Einstein-Weyl geometry. Colloq. Math. 81(1), 5-19 (1999)

10. Jelonek, W.: Higher dimensional Gray Hermitian manifolds. J. London Math. Soc. (2) 80(3), 729749 (2009)

11. Kobayashi, S.: Transformation groups in differential geometry. Springer, Berlin (1972)

12. Lichnerowicz, A.: Geometrie des groupes de transformations. Dunod, Paris (1958)

13. Madsen, B., Pedersen, H., Poon, Y., Swann, A.: Compact Einstein-Weyl manifolds with large symmetry group. Duke Math. J. 88 (1997), 407-434 (Corrigendum: Duke Math. J. 100 167-167 (1999))

14. Moroianu, A.: Lectures on Kähler Geometry. LMSST 69. Cambrige University Press, Cambrige (2007)

15. Pedersen, H., Swann, A.: Riemannian submersions, four-manifolds and Einstein-Weyl geometry. Proc. London Math. Soc 66(3), 381-399 (1993)

16. Pedersen, H., Swann, A.: Einstein-Weyl geometry, the Bach tensor and conformal scalar curvature. J. Reine Angew. Math. 441, 99-113 (1993)

17. Tod, K.P.: Compact 3-dimensional Einstein-Weyl structures. J. London Math. Soc. 45(2), 341-351 (1992)

18. Wang, J., Wang, M.Y.: Einstein metrics on $S^{2}$-bundles. Math. Ann. 310, 497-526 (1998) 